\title{
LA VIOLENCIA DE GÉNERO CONTRA LAS MUJERES EN YUCATÁN
}

\author{
Gender Violence against Women in Yucatan
}

\author{
Leticia Janet Paredes-Guerrero \\ Rodrigo Llanes-Salazar \\ Nayelli Torres-Salas \\ Alejandra Pamela España-Paredes
}

\begin{abstract}
Resumen: En el presente trabajo se analizan los tipos de violencia: doméstica, institucional y feminicida, usando el concepto de "violencia de género contra las mujeres", en combinación con las dimensiones de la violencia propuestas por Johan Galtung: interpersonal, estructural y cultural/simbólica, para demostrar que la violencia de género contra las mujeres en el estado de Yucatán, México, se ejerce no sólo en el hogar, sino también en las instituciones y en las representaciones colectivas, espacios en los que todavía se concibe a la mujer como un símbolo objeto, lo cual condiciona la aparición de conductas de violencia extrema que potencialmente desembocan en feminicidio.
\end{abstract}

Palabras clave: violencia de género, violencia doméstica, violencia estructural, violencia simbólica, violencia institucional, feminicidio.

Abstract: This paper analyzes different types of violence: domestic violence, institutional violence and femicide to show how gender violence against Yucatan's women is not only part of the domestic environment, but is also part of institutional environments and found in collective representations. To achieve this, the concept "gender violence against women" is used for analysis in combination with the spheres of violence as proposed by Johan Galtung: interpersonal or direct, structural and cultural/symbolic. Because women are objectified in these spheres, extreme violence and even feminicide are possible.

Keywords: gender violence, domestic violence, structural violence, symbolic violence, institutional violence, feminicide.

Leticia Janet Paredes Guerrero, doctora en Conocimientos y Cultura en América Latina por el Instituto de Pensamiento y Cultura en América Latina (IPECAL). Coordinadora del Grupo Universitario de Estudios de Género, de la Unidad de Ciencias Sociales del Centro de Investigaciones Regionales "Dr. Hideyo Noguchi” de la Universidad Autónoma de Yucatán, México. Temas de especialización: género, mujeres y política. Correo electrónico: guerrero@uady.mx.

Rodrigo Llanes Salazar, doctor en Ciencias Antropológicas por la Universidad Autónoma Metropolitana-Iztapalapa, México. Presidente del Colegio de Antropólogos de Yucatán, A.C., México. Temas de especialización: etnicidad, poder y violencia. Correo electrónico: rodrigo. llanes.s@gmail.com.

Nayelli Torres Salas, maestra en Género y Desarrollo por el Instituto de Estudios del Desarrollo de la Universidad de Sussex, Reino Unido. Asistente de Protección a la Infancia del Alto Comisionado de las
Naciones Unidas para los Refugiados. México. Temas de especialización: género, masculinidades, protección internacional a niñas y niños. Correo electrónico: nayelli.s.t@gmail.com.

Alejandra Pamela España Paredes, doctora en Educación por la Universidad del Sur, Campus Mérida, México. Profesora investigadora en la Unidad de Ciencias Sociales del Centro de Investigaciones Regionales Dr. Hideyo Noguchi de la Universidad Autónoma de Yucatán, México. Temas de especialización: género, políticas públicas y derechos humanos. Correo electrónico: espana.paredes@gmail.com.

Enviado a dictamen: 4 de enero de 2016. Aprobación: 5 de mayo de 2016.

Revisiones: 1 . 


\section{Introducción}

E n el presente trabajo nos ocupamos de mostrar la situación actual de la violencia de género ejercida en contra de las mujeres en el estado de Yucatán. Se abordan tres tipos de violencia: la familiar o doméstica, en su vertiente de violencia de pareja en el espacio del hogar — que es objeto de la mayoría de los trabajos académicos en la entidad y de las acciones de las organizaciones de la sociedad civil locales-, junto a la violencia institucional y la feminicida, que han sido menos trabajadas. Lo anterior nos ofrece una visión más completa de la violencia de género contra las mujeres más allá de las relaciones de pareja en el hogar, como producto del papel subordinado que ellas desempeñan en las relaciones sociales y de género imperantes en la sociedad yucateca, independientemente del estrato social al que pertenezcan.

Las condiciones de la violencia en contra de la mujer en Yucatán han dado pie a paradójicos registros por parte de la Organización para la Cooperación y el Desarrollo Económico (OCDE) y del Instituto Nacional de Estadística, Geografía e Informática (INEGI) a través de los resultados generados por la Encuesta Nacional sobre la Dinámica de las Relaciones en los Hogares 2014 (ENDIREH) que esta última institución coordinó. Así, en 2014 la entidad federativa fue catalogada por la primera institución como la más segura del país, sobre la base del índice de $7.35^{1}$ alcanzado en el reporte del estudio llamado "Para una vida mejor", en el que se destacaba la baja tasa de criminalidad y el bajo porcentaje de ciudadanos que consideraron el estado como inseguro; sin embargo, en ese mismo año los resultados de la ENDIREH situaron a Yucatán en el primer lugar en cuanto a violencia en las relaciones de pareja, ya que alcanzó el 48\%, dos puntos arriba de la media nacional.

Lo anterior explica por qué localmente se ha privilegiado el estudio de este tipo de violencia, que incluso influye en el accionar de las organizaciones de derechos humanos, cuyos integrantes han expresado frases como: "las mujeres aquí, en Yucatán, estamos más seguras en la calle que en la casa" o "estamos seguras en todos lados, menos en nuestros hogares". ${ }^{2}$

La invisibilización de la violencia de género en contra de las mujeres que ocurre más allá del ámbito privado, como son la institucional y la feminicida, obedece a su naturalización por el tipo de relaciones sociales prevalecientes en la entidad, que se refleja en omisiones en los marcos jurídicos locales relacionadas con el ejercicio de los derechos de las mujeres. En cuanto a los feminicidios, nos referimos a ellos como los homicidios cometidos contra mujeres por el odio que se siente hacia ellas debido al simple hecho de ser mujeres.

\section{La investigación}

En este artículo se retoma parte de los resultados de la investigación "Estudio regional sobre las fuentes, orígenes y factores que producen y reproducen la violencia contra las mujeres en la región sur de México, compuesta por los estados de Guerrero, Oaxaca, Chiapas, Campeche, Yucatán y Quintana Roo". Quienes participamos en Yucatán, realizamos una investigación cualitativa apoyada por fuentes documentales, entrevistas y trabajo grupal (Paredes, Llanes, Torres y España, 2012). En la primera parte se realizó una revisión exhaustiva de la bibliografía existente sobre la violencia contra las mujeres en Yucatán. En ella se da cuenta de que la temática comenzó a ser abordada a partir de la década de los noventa del siglo pasado. Se trata de estudios empíricos que abordan la violencia familiar, específicamente la de pareja — causas, prevalencia y características del agresor y la víctima-, y también se retomaron datos provenientes de documentos estadísticos y hemerográficos.

La violencia en los ámbitos laboral, institucional y comunitario, así como la violencia feminicida, fueron temas ausentes en los estudios académicos y en los documentos estadísticos, aunque sí se encontraron en la hemerografía. Por lo anterior, para ampliar lainformación acerca de la violencia en contra de las mujeres en dichos ámbitos también realizamos entrevistas y trabajo grupal con académicas y funcionarios públicos, así como con 
integrantes de organizaciones civiles que han trabajado sobre el tema de la violencia contra las mujeres.

\section{Organización del trabajo}

Este trabajo está compuesto por cinco partes. En la primera nos abocamos a definir qué entendemos por violencia de género contra las mujeres. En la segunda definimos la manera como utilizamos el planteamiento teórico del triángulo de la violencia propuesto por Johan Galtung (1969 y 1998), que adaptamos para analizar y superar la visión de la violencia como una problemática individual, para también entenderla como estructural y simbólica. En la tercera parte analizamos la violencia de género contra las mujeres ejercida por sus parejas en el espacio doméstico e identificamos las dimensiones de la violencia: estructural, simbólica e interpersonal o directa. En la cuarta parte nos ocupamos del feminicidio y de la violencia de género contra las mujeres en el espacio institucional, y en la quinta expresamos unos comentarios finales.

\section{Violencia de género contra las mujeres}

En 1993 la violencia contra la mujer se definió como:

[...] todo acto de violencia basado en la pertenencia al sexo femenino que tenga o pueda tener como resultado un daño o sufrimiento físico, sexual o psicológico para la mujer, así como las amenazas de tales actos, la coacción o la privación arbitraria de la libertad, tanto si se producen en la vida pública como en la vida privada (Asamblea General de las Naciones Unidas, 1993: 2).

En 1995, en la Cuarta Conferencia Mundial sobre la Mujer en Beijing se amplió la definición al señalarse que:

La violencia contra las mujeres se refiere a todo acto de violencia basado en el género que tiene como resultado posible o real un daño físico, sexual o psicológico, incluidas las amenazas, la coerción o la privación arbitraria de la libertad, ya sea que ocurra en la vida pública o en la privada (ONU,1996: 51).

Esta definición, al incorporar la expresión "todo acto de violencia basado en el género", dio pie a que poco más tarde se acuñara y usara el término "violencia de género", cuyo soporte deriva de los acuerdos a los que llegó la propia convención: "La violencia contra la mujer es una manifestación de las relaciones de poder históricamente desiguales entre mujeres y hombres, que han conducido a la dominación de la mujer por el hombre, a la discriminación contra la mujer y a la interposición de obstáculos contra su pleno desarrollo" (ONU, 1996: 52).

En este orden de ideas, Castro y Riquer (2010) señalan que la violencia de género tiene su origen, y persiste, en el conjunto de normas y valores sociales que sustentan la prevalencia o dominación masculina y la asimetría de poder entre hombres y mujeres. La existencia de marcadas normas de género que establecen roles socialmente aceptados para hombres y mujeres proporciona la justificación social para el uso de la violencia en la pareja cuando alguno de sus integrantes - mayoritariamente las mujeres- no cumple con los roles socialmente asignados, o de alguna manera transgrede las normas ante los ojos de su compañero (Castro y Riquer, 2012: 6). Para dichos autores, la violencia de género y la violencia contra la mujer son dos formas distintas de nombrar un mismo fenómeno.

En este trabajo se usa el término "violencia de género contra las mujeres" al considerar que el concepto, metodológicamente, integra dos partes: la primera, "violencia de género", pone énfasis en todo acto que, por diferencia de género, puede ocasionar daño o sufrimiento a las mujeres; la segunda, "contra la mujer", tiene como característica central — de acuerdo con la definición de 1993 - el "sexo femenino", como el cuerpo en el que se perpetra el daño físico, emocional y sexual.

Susana Velázquez afirma que la "violencia de género" es más que la descripción del maltrato físico, del abuso emocional, del incesto y de la violación, pues debe ofrecerse un valor explicativo cuyo punto central sea la 
noción de género, porque la violencia se basa y se ejerce en y por la diferencia social y subjetiva entre los sexos y por las relaciones de poder (Velázquez, 2010: 28-29).

María Rivera sostiene que "la violencia contra las mujeres no es violencia de género". Para ella, el punto central de "la violencia contra la mujer" se encuentra en el no reconocimiento de la autoridad de las mujeres en las relaciones sociales que establecen, y la ejercen típicamente los hombres (Rivera, 2001: 1).

Los planteamientos anteriores nos llevan a definir y usar el concepto de "violencia de género contra las mujeres" para explicar que en Yucatán ellas viven la violencia como un problema estructural y relacional en la medida en que hay relaciones de dominio y de asimetría de género culturalmente construidas que les ocasionan daño o sufrimiento, que simbólicamente se encuentran normalizadas como una práctica de género y se manifiestan en el cuerpo de las mujeres.

\section{El triángulo de la violencia}

Creemos importante relacionar las consideraciones anteriores sobre el concepto de "violencia de género contra las mujeres" con los planteamientos teóricos del sociólogo noruego Johan Galtung (1969 y 1998). De acuerdo con él, la violencia es una relación social que tiene como fin la provocación de daño o sufrimiento y la limitación de las potencialidades presentes o futuras de un individuo o una colectividad. Galtung distingue tres dimensiones de la violencia, que llamaremos triángulo de la violencia de género: la "directa" o "interpersonal", es decir, aquella que se presenta de manera directa entre dos o más actores concretos; la "estructural" o "indirecta", que no es ejercida por un actor específico, sino que se genera a partir de las condiciones sociales y que tiene como fundamento la distribución desigual de los recursos, y la "cultural", que es la que se basa en representaciones y elementos simbólicos sobre los sujetos. Estas tres dimensiones de la violencia, argumenta Galtung, forman un triángulo en cuyo vértice superior se encuentra la violencia directa o interpersonal que, como la punta de un iceberg, es la parte más visible del fenómeno de la violencia. No obstante, en la base de dicho triángulo se encuentran la violencia estructural y la cultural que, siguiendo con la metáfora del iceberg, permanecen sumergidas en lo profundo de la sociedad y, por lo tanto, resultan poco visibles para los sujetos, aunque pueden ser visibilizadas a través de la investigación y de la acción política (Galtung, 1969: 167-191).

En principio, la propuesta teórica de Galtung es útil para analizar cualquier tipo de violencia. Para el estudio de la violencia de género contra las mujeres, retomamos su modelo a la luz de las discusiones sobre género y sobre violencia de género. En este sentido, la violencia directa o interpersonal - la que ocupa la punta del triángulo- es la que se manifiesta como daño o sufrimiento que las mujeres experimentan en su cuerpo, como emociones o bienestar material; así, se habla de violencia física, emocional, económica y sexual. Es la parte visible de la violencia que ha concentrado el interés de los trabajos realizados en el estado de Yucatán.

Para el estudio de la base del triángulo - la violencia estructural y la cultural-, retomamos el análisis de la desigualdad de género basada en prescripciones, asignaciones sociales, normas y roles que colocan a hombres y mujeres en condiciones, posiciones y valoraciones asimétricas, lo que da pauta para la "violencia estructural" y nos dirige al concepto de "violencia simbólica" del sociólogo Pierre Bourdieu (2000), el cual se refiere a cómo los esquemas de percepción de los agentes dominantes son interiorizados también por los dominados de forma tal que la violencia y la dominación, que siempre son producto de procesos sociales e históricos, aparecen como algo natural y normal para dominantes y dominados.

De ambos conceptos queremos enfatizar que, en un nivel estructural y simbólico, los hombres aparecen como "sujetos productivos" y "proveedores", cuyo espacio por excelencia es el ámbito público, mientras que las mujeres son conceptualizadas como "objeto" para el hombre y su espacio es el ámbito privado. En dicho sistema, el hombre ocupa una posición más valorada que la mujer, ${ }^{3}$ por lo que nos encontramos con relaciones desiguales de poder en las que los hombres controlan o 
buscan controlar los recursos más significativos y toman las decisiones sobre ellos. De este modo, la violencia de género contra las mujeres no se trata de una serie de casos aislados, producto únicamente de tensiones, celos o conflictos situacionales, sino que su base se encuentra en un sistema de sexo/género marcado por desiguales relaciones de género y de poder que alimentan la violencia simbólica.

Los planteamientos anteriores nos llevan a explicar el caso yucateco utilizando el triángulo de la violencia de forma invertida, es decir, analizando primero las dimensiones de la violencia estructural y simbólica y, por último, de la directa o interpersonal, lo cual nos permite un análisis relacional que hace patente que la violencia de género contra las mujeres yucatecas se encuentra presente, y de manera importante, no sólo en el hogar, sino también en las instituciones y en las representaciones colectivas, que todavía conciben a la mujer como un símbolo objeto, lo que condiciona la aparición de conductas de violencia extrema que pueden desembocar en feminicidio.

\section{Violencia de género contra las mujeres: relaciones de pareja}

\section{Violencia estructural}

La condición y la posición de desigualdad, dominación, desventaja y asimetría de género de las mujeres en Yucatán en los ámbitos social, educativo, laboral, político y económico nos dan cuenta de la violencia estructural que existe contra ellas. Los datos estadísticos del censo del INEGI de 2010 indican que Yucatán tenía en ese año una población de 1955577 habitantes, de los que 992244 eran mujeres y 963333 hombres. Según la misma fuente, en el estado había 503106 hogares registrados: 390167 con jefatura masculina y 112939 con jefatura femenina; es decir, uno de cada cinco hogares en Yucatán estaba comandado por una mujer (INEGI, 2010: 3). A los datos antes mencionados hay que agregar que, de los 106 municipios de Yucatán, 10 presentaban un muy alto grado de marginación, 23 un alto grado, 68 un grado medio, 4 un bajo grado, y sólo el municipio de Mérida poseía un grado muy bajo de marginación (CONAPO, 2011).

La desigualdad económica entre hombres y mujeres se observaba en los ingresos provenientes del trabajo remunerado porque, mientras las mujeres en el estado ganaban en promedio 4521 dólares per cápita, los hombres percibían 10065 (INEGI, 2010: 2). Asimismo, la ENDIREH (2011) indica que eran las mujeres urbanas y aquellas con ingresos entre los 3000 y 9000 pesos mensuales las más vulnerables a la violencia, por lo que la pobreza, particularmente la feminización de la pobreza asociada con el trabajo doméstico y con la doble, e incluso triple, jornada laboral, ${ }^{4}$ era una condición que generaba asimetría de género.

El INEGI también dio a conocer que 201524 mujeres revelaron que habían sido víctimas de violencia en el año 2015, de las cuales 112579 reportaron estar casadas y 73882 solteras (Cárdenas, 2015: 5). Ciertamente, el hecho de encontrar casos de violencia de género tanto en las clases altas y en sectores con educación superior en el ámbito urbano, ${ }^{5}$ como entre los sectores más marginados y pobres del estado, parece confirmar la idea de que la violencia de género contra las mujeres en Yucatán afecta a mujeres de todos los estratos sociales.

El educativo es otro ámbito en el que se puede apreciar la desigualdad de género; en 2010, el porcentaje total de analfabetas en el estado era del $9.23 \%-7.78 \%$ de hombres y $10.62 \%$ de mujeres- ${ }^{6}$ (INEGI, 2010: 5). Esta condición desigual en la educación de las mujeres, de acuerdo con los datos proporcionados por el Instituto de Equidad y Género de Yucatán (IEGY), contribuyó al ascenso de la violencia sexual durante el período 2003-2006, siendo las mujeres de entre 50 y 54 años las que se ubicaron en el primer lugar (IEGY, 2008: 52).

La representación numérica de las mujeres en la política también muestra desigualdad pues, en cuanto al número de diputados estatales, según los resultados de las tres últimas elecciones que tuvieron lugar en 2010, 2012 y 2015, en los primeros dos procesos las legislaturas correspondientes fueron integrados por veinte hombres y cinco mujeres, por lo que las mujeres ocuparon solamente el $20 \%$ de las curules. 
En la legislatura de 2015 a 2018 incrementó de forma importante la participación política de las mujeres, al llegar a nueve las diputadas, lo que representa el $36 \%$, porcentaje todavía lejano a la paridad (Paredes, 2015). Estos números pudieran hacernos suponer que las mujeres ocupan puestos de representación para la toma de decisiones; sin embargo, nos cuestionamos si su representación ha sido sustantiva porque no ha contribuido a la construcción de una agenda legislativa con equidad de género, mientras que sí se han experimentado retrocesos legislativos, como la desaparición de la Comisión de Equidad de Género del Congreso (Paredes, 2013).

Para las mujeres, la condición étnica es un elemento estructural que las coloca en condición de discriminación y subordinación. ${ }^{7}$ En Yucatán, el $62.69 \%$ de la población de tres años y más se reconoce como perteneciente a un pueblo indígena. En 2010, eran mayas el $49.66 \%$ de las mujeres yucatecas y el 23.16\% de las mujeres de la ciudad de Mérida (Equipo Indignación, 2013). De las hablantes de una lengua indígena casadas o unidas, el 39\% había sufrido violencia a lo largo de su relación. En 2011 Yucatán registró el mayor porcentaje de mujeres hablantes de alguna lengua indígena violentadas por familiares (16.6\%) y ocupó uno de los primeros lugares entre los estados con mayor porcentaje de mujeres con estas características que declararon alguna clase de agresión en lugares públicos (18.6\%). Asimismo, según el IEGY, las mujeres mayas sufren una doble discriminación por ser mujeres y de origen maya; por ello, han sentido la desigualdad de trato y han tenido acceso limitado a niveles educativos o laborales debido a su condición étnica, genérica y de clase (IEGY, 2008: 28).

Las condiciones de las mujeres yucatecas descritas anteriormente nos dan cuenta de que en la estructura confluyen desigualdades, subordinación, discriminación, dominio y asimetría en los ámbitos económico, de clase, educativo, político y étnico que, al relacionarse, contribuyen al desarrollo de una violencia estructural que coloca a las mujeres en posiciones vulnerables para la manifestación de otros tipos de violencia.

\section{Violencia simbólica}

La violencia simbólica (Bourdieu, 2000) tiene como característica que logra imponer significados como legítimos, lo cual invisibiliza las relaciones de dominio y logra que los agentes dominantes sean interiorizados también por los dominados, de forma tal que la violencia y la dominación, que siempre son producto de procesos sociales e históricos, aparecen como algo natural y normal para dominantes y dominados.

Al respecto, la antropóloga Yolanda Oliva (2004) documentó la violencia verbal en la pareja como una expresión de violencia simbólica, al dar cuenta de que existen expresiones legitimadas que promueven sexismo y dominio, y que a la vez normalizan, naturalizan y justifican la violencia. Algunas expresiones de este tipo, señala la autora, son: "porque te quiero, te aporreo", "si a la mujer la maltrata su marido es porque algo habrá hecho", "el hombre tiene el derecho de maltratar a su esposa: para eso es su marido", "una mujer solamente es maltratada cuando el hombre tiene un problema", "a las mujeres les gusta que les peguen", "las mujeres golpeadas son masoquistas", "si la mujer tiene hijos, debe pensar primero en ellos y es preferible que se quede con el marido" o "lo más importante es que la familia esté junta" (Oliva, 2004: 38-40).

La violencia simbólica como la legitimación del dominio masculino también se expresa en prácticas, conductas y representaciones que promueven y reproducen percepciones como las siguientes: 1) las mujeres deben resistir y aguantar el maltrato como parte de su "misión" de mujer y madre, 2) los hombres tienen derecho a ejercer la violencia porque "sus mujeres" son de su propiedad, o 3) las mujeres deben aguantar la violencia porque antes de pensar en ellas deben considerar a su familia (Rosado, 2009).

Este tipo de violencia adquiere un alto nivel de gravedad en las mujeres jefas de familia pues, al tener esa posición, cuentan con mayor independencia económica, la cual presupone una mayor autonomía en la toma de decisiones, lo que representa una amenaza a la práctica de los roles de género tradicionales en los hogares, así como al dominio masculino (Castro, 2006; Goyri y 
Moreno, 2009; Oliva, 2004; Paredes, 2005; Villagómez, 2005). Es decir, se trata de un "síntoma de la fractura de la domesticidad" (Castro y Riquer, 2012: 11) y los hombres buscan disciplinar a las mujeres - autónomas o rebeldes - a través de la violencia (Femenías y Soza, 2009), para lo cual recurren a la violencia simbólica con el propósito de legitimar que el hogar y la familia son los espacios de las mujeres, mientras que el del trabajo corresponde a los hombres. Por lo anterior, la responsabilidad de cualquier situación conflictiva en las relaciones de pareja o en el hogar recae en las mujeres trabajadoras, ya que ocupan un rol de género y una posición al interior de la familia no "natural".

\section{Violencia interpersonal}

La violencia que Galtung conceptualiza como interpersonal o directa es aquella cuyas manifestaciones son más explícitas. La ENDIREH distingue cuatro tipos de violencia: la física, la emocional, la económica y la sexual. Según dicha encuesta, en 2006 el 30.5\% de las mujeres encuestadas en el estado declararon haber sufrido algún tipo de violencia. Si bien no siempre son tan claramente delimitables las violencias física, emocional, económica y sexual, ${ }^{8}$ el tipo de violencia hacia las mujeres en los hogares en Yucatán más registrado en la encuesta fue la emocional, con un 21.8\%; le seguía la económica, con $16.8 \%$, luego la física, con $8.7 \%$, y por último la sexual, con un $5.6 \%$. Tomando como base las encuestas de la ENDIREH, el IEGY ha señalado que los resultados aún no permiten generar los perfiles de agresores y víctimas (IEGY, 2008: 1).

Los estudios sobre violencia directa o interpersonal en Yucatán se han centrado en el espacio doméstico y han dado cuenta de que la familia no es solamente un espacio armónico y de refugio, sino que en él también se inscriben relaciones de poder y desigualdad, tensiones, conflictos y violencia; del mismo modo, sabemos que la violencia familiar no es un asunto privado, sino que también es público y político. Igualmente, somos más conscientes de que la violencia, en sus diversas expresiones y dimensiones, suele ser naturalizada y normalizada de forma tal que muchas veces no es percibida ni reconocida como violencia, aun cuando ha sido ampliamente estudiada y se han establecido políticas públicas para su atención.

\section{Violencia de género contra la mujer institucional y feminicida}

En el apartado anterior dimos cuenta de que para comprender la violencia de género contra las mujeres es necesario establecer la relación entre la estructura, el símbolo y lo interpersonal, pero también señalamos que la violencia interpersonal en el espacio doméstico, específicamente en las relaciones de pareja, es la que más se ha visibilizado para el caso yucateco a través de diversos estudios y acciones concretas de política pública. Ahora nos ocuparemos de dar cuenta de la violencia institucional y feminicida.

\section{Violencia institucional}

Entendemos como violencia institucional las omisiones y negligencias de las instituciones para garantizar el ejercicio de los derechos de las mujeres, que afecta tanto al ámbito doméstico como al público. No se trata necesariamente de un tipo de violencia directa o interpersonal, sino más bien es de tipo estructural porque surge a partir de la desigualdad de oportunidades, entre otros puntos por la distribución de los recursos de forma no igualitaria, y se trata de violencia cultural/simbólica en la medida en que el dominio sobre las mujeres se normaliza y legitima.

Este tipo de violencia institucional de género contra las mujeres en el estado se observa también en el acceso a la justicia por parte de las mujeres víctimas de violencia y en cómo ésta se imparte en sus casos concretos (Equipo Indignación, 2013). A pesar de la existencia de instituciones y leyes que deben prevenir, atender y erradicar la violencia de género, diversas especialistas y funcionarias públicas relataron que es frecuente que, cuando una mujer denuncia a su pareja o familiar por actos de violencia, los funcionarios que las atienden les formulan preguntas tales como: "ipara qué denuncias si es tu marido?”, "ipor qué denuncias a tu marido?, ¿ahora 
quién te va a mantener"? Con expresiones como estas ejercen violencia simbólica al reproducir los esquemas del hombre como proveedor y de la mujer que debe poner en primer lugar a su familia o a su pareja antes que su integridad como ser humano.

Además, la violencia simbólica, la revictimización y la falta de atención por parte de las instituciones, como la Fiscalía General del Estado, puede tener consecuencias incluso fatales para las mujeres, tal como ha documentado la organización civil Equipo Indignación (2013 y 2014). En palabras de dicha organización: "en Yucatán, [tanto el poder] Ejecutivo [como el] Legislativo han exhibido una gran tolerancia frente a la violencia de género, lo que constituye violencia institucional, favorece la revictimización y la impunidad", ya que "la lenta y despreocupada administración de justicia (más preocupada por formalismos que por las víctimas) resulta ser tan dañina para las víctimas como las violaciones primarias a sus derechos humanos" (2013: 43). Esto es una muestra de violencia estructural de género, ya que la organización misma de la sociedad y el funcionamiento de sus instituciones producen sufrimiento a las mujeres.

Un ejemplo de lo anterior es el caso de Janie Rosaura Salazar Pérez de la comisaría San José Tzal, al sur de Mérida. A pesar de un largo historial de agresiones y malos tratos por parte de su esposo, al que ya había denunciado, Janie Rosaura no lograba que las instituciones dieran seguimiento a su caso. Tanto la Fiscalía del Estado como la Procuraduría de la Defensa del Menor y la Familia le propusieron que se reconciliara con su esposo y nunca se dictaron medidas cautelares. Otra mujer, Leydi Pech, convenció a Janie Rosaura de que abandonara a su esposo y las dos vivieron alrededor de un mes juntas. Sin embargo, el l de agosto de 2011 Janie Rosaura fue asesinada por su esposo junto con sus dos hijos con una pistola calibre 32 .

Los casos anteriores ilustran cómo la violencia institucional puede manifestarse en las tres dimensiones de violencia. Es violencia directa porque un funcionario discrimina a una mujer por el hecho de serlo, es estructural porque la institución carece de mecanismos eficaces para evitar la discriminación, y es simbólica porque se legitima que ciertas personas sean valoradas de manera desigual en razón de ciertos marcadores sociales.

Pero la violencia institucional se agrava cuando las mujeres son de la etnia maya, ya que a las prácticas de género existentes se suma su condición étnica, por lo que les resulta mucho más difícil acceder a la justicia. En este sentido, integrantes de la organización defensora de derechos humanos Equipo Indignación nos dijeron:

Las mujeres mayas viven la violencia de otra forma. Se enfrentan a muchos más obstáculos. No están reconocidos los derechos del pueblo maya: su primera instancia para acceder a la justicia es quien hace la justicia en su propia comunidad y en Yucatán los derechos del pueblo maya están muy mal reconocidos y han vulnerado el acceso del pueblo maya de hacer justicia. Por ejemplo, las mujeres mayas no pueden declarar en su lengua. Se enfrentan a un sistema de justicia que no es propio de ellas como mujeres mayas. Se enfrentan a un sistema de justicia corrupto, negligente y a otra cosmovisión (personal de Equipo Indignación).

\section{Violencia feminicida}

Otra de las ausencias en materia de investigación y acción sobre la violencia hacia las mujeres, que ciertamente es una de las más alarmantes, es la violencia feminicida. Podemos definir el feminicidio como el crimen de odio hacia las mujeres por el hecho de serlo. Se trata de una expresión extrema de violencia simbólica hacia las mujeres basada en el sistema sexo/ género: sus cuerpos son reducidos a meros objetos. "El cuerpo es visto como una cosa cualquiera", expresaba Adelaida Salas, responsable estatal del Observatorio Ciudadano Nacional de Feminicidios. De acuerdo con dicho observatorio, existen 44 casos de feminicidio registrados en Yucatán de 2007 a 2012; 24 de ellos fueron cometidos por las parejas o por familiares de las víctimas, principalmente por los esposos. Si bien en septiembre de 2012 el feminicidio fue incorporado como delito al Código Penal del estado de Yucatán, 
fue en 2014 cuando se incluyó como "delito grave" (Equipo Indignación, 2014). Mientras tanto, en 2013 se registraron trece casos, y diez más en 2014.

Más allá de las cifras, las académicas y activistas destacan el caráctercada vez más cruel delosfeminicidios en el estado. La responsable del Observatorio Ciudadano Nacional de Feminicidios expresó que:

Tenemos 44 casos de feminicidios, muy crueles. Algunas tiradas, con abrelatas en la vagina. Casos terribles. 44 en todo el estado. Ha habido en Mérida, ha habido en clase alta, clase baja, ha habido en el medio rural, ha habido en jóvenes. Hay factores de alcoholismo, hay quien mata por celos, hay la prostituta que la mataron. Tenemos de todo, no hay nada que te diga específico. Es diverso el universo. En Yucatán no hay un grupo de mujeres más vulnerable ante el feminicidio (entrevista responsable del Observatorio Ciudadano Nacional de Feminicidios).

El testimonio anterior no sólo da cuenta del carácter sumamente cruel de los feminicidios en Yucatán, sino que también acentúa la idea de que afecta por igual a las diversas clases, sectores o grupos de edad. Todas las mujeres son igual de vulnerables. Consideramos este tipo de violencia como directa, y se encuentra íntimamente relacionada con la estructural y la simbólica. Los casos que presentamos a continuación de feminicidio en el ámbito rural contra mujeres mayas o migrantes, todas ellas menores de edad, nos dan cuenta de ello.

En años recientes se han encontrado cadáveres de mujeres víctimas de feminicidio en el municipio de Maxcanú, ubicado en la frontera de Yucatán con Campeche, que cuenta con un alto porcentaje de maya hablantes. Es el caso de la joven de 16 años Reina Alejandra Polanco Uicab, cuyo cuerpo carbonizado fue encontrado en un monte el 24 de enero de 2012. A partir de ese acontecimiento, se ha puesto de manifiesto que hay un elevado número de jóvenes desaparecidas en el municipio - al menos se reportan diez desaparecidas, todas ellas jóvenes de entre 14 y 24 años- e incluso ha comenzado a hablarse de la existencia de una red de prostitución infantil que opera en cantinas del municipio, como las llamadas El Venado, El Plantel y El Chaparral. De acuerdo con notas de prensa, los clientes provienen principalmente de las ciudades de Mérida y Campeche y el negocio está controlado por un conocido taxista del lugar, quien las "engancha" en las comisarías ${ }^{9}$ del municipio ofreciéndoles trabajo bien remunerado. En la cantina El Chaparral se ha instalado una suerte de hotel donde los clientes pagan 400 o 450 pesos por servicios sexuales con las menores (Chan, 2012; Sauri, 2012).

El caso anterior ilustra la violencia estructural de género y la interseccionalidad, en la que las mujeres jóvenes, maya hablantes y del ámbito rural son las más vulnerables y las más propensas a convertirse en víctimas de comercio sexual y de delitos como desaparición y feminicidio. La violencia simbólica opera cuando las autoridades estatales encubren el feminicidio considerándolo como "crimen pasional", producto de los "celos" y de una "relación tormentosa, y reducen el acto al ámbito familiar sin reconocer las formas de organización social — como el comercio sexual - ni el papel de las instituciones en dichos delitos.

\section{Consideraciones finales}

A partir de lo expuesto, puede afirmarse que los estudios sobre violencia contra la mujer en Yucatán únicamente contemplan la dimensión interpersonal o directa de la violencia en el espacio doméstico, específicamente en las relaciones de pareja, y la reducen al espacio privado, lo cual limita la explicación del fenómeno en la entidad. Por ello, ampliamos la mirada y señalamos que la violencia de género en contra de las mujeres está presente tanto en el espacio privado como en el público; sin embargo, la violencia institucional y la feminicida, expresadas en el espacio público, se han naturalizado debido al tipo de relaciones sociales basadas en la desigualdad, lo cual se refleja en una omisión de los marcos normativos.

Un planteamiento importante para entender la violencia vivida por las mujeres yucatecas fue la definición del concepto "violencia de género contra 
las mujeres", ya que nos permitió dar cuenta de que la violencia es resultado de relaciones desiguales de poder entre los géneros que causan daño en el cuerpo de las mujeres.

Relacionar el concepto "violencia de género contra las mujeres" con el planteamiento teórico del sociólogo Galtung, quien propone tres dimensiones para abordar la violencia -interpersonal, estructural y cultural o simbólica-, lo que se denomina "triángulo de la violencia”, enriqueció nuestro análisis, pues utilizar este triángulo de forma invertida — es decir, analizando primero las dimensiones de la violencia estructural y simbólica y por último la directa o interpersonal- nos permitió efectuar un análisis relacional que hace patente que la violencia de género contra las mujeres yucatecas se encuentra presente y de manera importante, no sólo en el hogar, sino también en las instituciones y en las representaciones colectivas, en las que todavía se concibe a la mujer como un símbolo objeto, que condicionan la aparición de conductas de violencia extrema que pueden desembocar en feminicidio.

Por último, los datos estadísticos y hemerográficos, así como los obtenidos en entrevistas, nos permitieron dar cuenta de que la violencia de género contra las mujeres es relacional entre la estructura, el símbolo y lo interpersonal, elementos presentes en la sociedad yucateca tanto en el espacio privado, como en el público.

\section{Notas}

1 Ver: http://www.elfinanciero.com.mx/mas/enfoques/ yucatan-sobresale-en-empleo-y-seguridad-ocde.html y http://www.milenio.com/policia/Mexico-ultimolugar-OCDE-seguridad_0_323967608.html. El índice de la OCDE se puede consultar en: http://www. oecdbetterlifeindex.org/es/.

2 Sobre la idea del hogar como el lugar menos seguro para las mujeres, ver Hearn (1998); tómese en cuenta también que, como observa Contreras Urbina: "es a través de la familia de origen donde primordialmente se ponen en práctica la legitimidad y la condena de la violencia contra la mujer" (2008: 73).
3 La antropóloga francesa Françoise Héritier (1996) se refiere a esta relación de desigualdad como una "valencia diferencial de los sexos" en la que, en un nivel estructural, lo masculino es valorado como superior a lo femenino.

${ }^{4}$ Sobre la relación entre la feminización del trabajo y de la pobreza, el neoliberalismo y la violencia hacia las mujeres, ver Zúñiga (2008).

${ }^{5}$ Considérese, por ejemplo, el caso de acoso de un investigador del Centro de Investigación Científica de Yucatán (CICY) (Paredes et al., 2012: 71).

${ }^{6}$ En términos nacionales, Castro y Riquer anotan que: "en la ENDIREH 2011 se confirma la enigmática tendencia detectada desde 2003, en el sentido de que las prevalencias más altas para la violencia física, emocional y económica se concentran entre las mujeres con secundaria incompleta [...] hasta ahora no ha sido posible identificar en las variables que caracterizan a las mujeres alguna posible explicación a este patrón" (2012: 34).

${ }^{7}$ La interseccionalidad política subraya el hecho de que las mujeres de color están situadas en al menos dos grupos subordinados que frecuentemente persiguen agendas políticas distintas e incluso en conflicto (Crenshaw, 1991: 1251-1252).

${ }^{8}$ En un trabajo reciente, los diseñadores de la ENDIREH han criticado esta clasificación señalando que no siempre son tipos claramente distinguibles (Castro y Riquer, 2012).

9 Comisarías y subcomisarías son comunidades que pertenecen a los municipios pero se encuentran fuera de las cabeceras municipales. De acuerdo con el artículo 12 de la Ley de Gobierno de los Municipios del Estado de Yucatán (2012) los conceptos se definen como sigue: "IV.- Comisaría, es el núcleo de población con censo no menor a los quinientos vecinos" y "V.- Sub-comisaría, el núcleo de población con censo inferior a quinientos vecinos".

\section{Referencias bibliográficas}

Asamblea General de las Naciones Unidas (1993). Declaración sobre la eliminación de la violencia 
contra la mujer. Resolución 48/104. Disponible en: http://www.ohchr.org/SP/ProfessionalInterest/ Pages/ViolenceAgainstWomen.aspx (consultado el 30 de junio de 2012).

Bourdieu, Pierre (2000). La dominación masculina. Barcelona: Anagrama.

Castro Gamboa, Rita Concepción (2006). Incidencia de la violencia familiar en el comportamiento suicida de la mujer en Yucatán. Tesis de Maestra en Ciencias Antropológicas opción Antropología Social. Mérida: Universidad Autónoma de Yucatán.

Castro, Roberto y Florinda Riquer (2003). "La investigación sobre violencia contra las mujeres en América Latina: entre el empirismo ciego y la teoría sin datos". En Cadernos de Saúde Pública, vol. 19, núm. 1, pp. 135-146.

Castro, Roberto y Florinda Riquer (2012). "Claroscuros en el conocimiento sobre la violencia contra las mujeres". En Irene Casique y Roberto Castro (coords.), Retratos de la violencia contra las mujeres en México. Análisis de resultados de la Encuesta Nacional sobre la Dinámica de las Relaciones en los Hogares, 2011. México: Instituto Nacional de las Mujeres, pp. 9-35.

Cárdenas, Israel (2015). "Buscan el empoderamiento de las féminas: Más de 200 mil mujeres yucatecas han sido víctimas de algún tipo de agresión, según reciente censo del INEGI. Persiste violencia de género". En Milenio Novedades, 23 de octubre, p. 6.

Contreras Urbina, Juan Manuel (2008). "La legitimidad social de la violencia contra las mujeres en la pareja. Un estudio cualitativo con varones en la ciudad de México". En Roberto Castro e Irene Casique (eds.), Estudios sobre cultura, género y violencia contra las mujeres. México: Universidad Nacional Autónoma de México/Centro Regional de Investigaciones Multidisciplinarias, pp. 4l-79.

Crenshaw, Kimberlé (1991). "Mapping the Margins: Intersectionality, Identity Politics, and Violence against Women of Color". En: Stanford Law Review, vol. 43, núm. 6, pp. 1241-1299.

Chan Caamal, David (2012). "Habría una red de prostitución infantil tras un homicidio". En Observatorio de Violencia Social y Género, 31 de enero. Disponible en http://www.ovsgyucatan.uady.mx/avisosynoticias. html (consultado el 30 de junio de 2012).

Equipo Indignación (2013). La casta Susana. Mujeres sin justicia en Yucatán. Mérida: Equipo Indignación

Equipo Indignación (2014). "Feminicidio, al fin grave en Yucatán”. En Indignación, 20 de marzo. Disponible en: http://indignacion.org.mx/2014/03/20/feminicidiodelito-grave/ (consultado el 26 de marzo de 2014).

Femenías, María Luisa y Paula Soza Rossi (2009). "Poder y violencia sobre el cuerpo de las mujeres". En Sociologías, año 1l, núm. 21, pp. 42-65.

Galtung, Johan (1969). "Violence, Peace, and Peace Research". En Journal of Peace Research, vol. 6, núm. 3, pp. 167-191.

Galtung, Johan (1998). Tras la violencia, 3R: reconstrucción, reconciliación, resolución. Afrontando los efectos visibles e invisibles de la guerra y la violencia. Bilbao: Bakaeaz/ GernikaGogratuz.

Goyri Ceballos, Jaime Abraham y Sergio Andrés Moreno Cabrera (2009). Ideas y reflexiones del trabajo con hombres en atención a la violencia intrafamiliar en Mérida, Yucatán. Mérida: Instituto Nacional de las Mujeres/Gobierno Federal/Instituto para la Equidad de Género en Yucatán/Ayuntamiento de Mérida/Kóokay, Ciencia Social Alternativa A.C.

Hearn, Jeff (1998). The Violence of Men: How Men Talk about and How Agencies Respond to Men's Violence to Women. Londres: Sage.

Héritier, Françoise (1996). Masculino/femenino. El pensamiento de la diferencia. Barcelona: Ariel.

INEGI (Instituto Nacional de Estadística y Geografía) (2010). Las mujeres en Yucatán. Estadísticas sobre desigualdad de género y violencia contra las mujeres. México: Instituto Nacional de Estadística y Geografía/Fondo de Desarrollo de las Naciones Unidas para la Mujer.

IEGY (Instituto para la Equidad de Género en Yucatán) (2008). Análisis de la violencia contra las mujeresen Yucatán, 2006. Mérida: IEGY.

Oliva Peña, Yolanda (2004). La construcción social de la violencia de género en las relaciones de pareja en Caucel, Yucatán. Tesis de Maestría en Ciencias Antropológicas con la opción Antropología Social. Facultad de 
Ciencias Antropológicas-Universidad Autónoma de Yucatán, Mérida.

ONU (1996). Informe de la Cuarta Conferencia Mundial sobre la Mujer. Beijing, 4 a 15 de septiembre de 1995. Nueva York: ONU. Publicación de las Naciones Unidas A/ CONF.177/20/Rev. 1.

Paredes Guerrero, Leticia (2005). "Acuerdos internacionales y violencia de género" [documento inédito].

Paredes Guerrero, Leticia (2013). "El movimiento feminista en la construcción de la agenda legislativa de equidad y género en Yucatán”. En M. Hernández y J. Rodríguez (coords.). Guijarros: avances y retrocesos de la participación política de las mujeres en México. México: Porrúa.

Paredes Guerrero, Leticia (2015). "La participación política de las mujeres en Yucatán: de la lucha por la ciudadanía al ejercicio de ser ciudadanas (19001915)". En F. Sánchez (coord.), Actualización de la enciclopedia yucatanense, t. 13. Mérida: CONACULTA/ SEDECULTA/Gobierno del Estado de Yucatán.

Paredes, Leticia, Rodrigo Llanes, Nayelli Torres y Pamela España (2012). Estudio sobre las fuentes, orígenes y factores que producen y reproducen la violencia contra las mujeres en Yucatán [documento inédito].

Rivera Garretas, María-Milagros (2001). "La violencia contra las mujeres no es violencia de género". En
Duoda. Revista d'Estudis Feministes, Revista de Estudios Feministas, núm 21.

Rosado y Rosado, María (2009). "Violencia de género en las relaciones de pareja y sus efectos en los hijos". En Leticia Paredes Guerrero y Georgina Rosado Rosado (coords.), Familia y relaciones de género en Yucatán. Mérida: Universidad Autónoma de Yucatán, pp. 203 218.

Sauri Riancho, Dulce María (2012). "Vida sin violencia. En los zapatos de Reina Alejandra”. En Dulce María Sauri Riancho [blog], 1 de febrero. Disponible en http:// dulcesauri.blogspot.mx/2012/02/vida-sin-violenciaen-los-zapatos-de.html (consultado el 30 de junio de 2012).

Velázquez, Susana (2010). Violencias cotidianas, violencias de género. Escuchar, comprender, ayudar. Buenos Aires, Barcelona, México: PAIDOS.

Villagómez Valdés, Gina (2005). Familia y violencia. Políticas de atención a la violencia doméstica en Mérida. Mérida: Ayuntamiento de Mérida 2004-2007.

Zúniga Elizalde, Mercedes (2008). "Violencia en el trabajo. La cultura de la dominación de género". En Roberto Castro e Irene Casique (eds.), Estudios sobre cultura, género y violencia contra las mujeres. México: Universidad Nacional Autónoma de México/Centro Regional de Investigaciones Multidisciplinarias, pp. 173-196. 